\title{
Fission studies at IGISOL/JYFLTRAP: Simulations of the ion guide for neutron-induced fission and comparison with experimental data
}

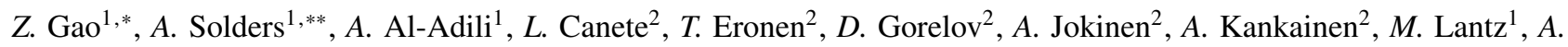 \\ Mattera $^{1}$, I.D. Moore ${ }^{2}$, D.A. Nesterenko ${ }^{2}, H$. Penttilä ${ }^{2}, I$. Pohjalainen $^{2}, V$. Rakopoulos ${ }^{1}, S$. Rinta-Antila ${ }^{2}, M$. Vilén ${ }^{2}$, and \\ S. Pomp ${ }^{1}$
}

${ }^{1}$ Uppsala University, BOX 516, 75120 Uppsala, Sweden

${ }^{2}$ University of Jyväskylä, P.O.Box 35 , 40014 Jyväskylä, Finland

\begin{abstract}
For the production of exotic nuclei at the IGISOL facility, an ion guide for neutron-induced fission has been developed and tested in experiments. Fission fragments are produced inside the ion guide and collected using a helium buffer gas. Meanwhile, a GEANT4 model has been developed to simulate the transportation and stopping of the charged fission products. In a recent measurement of neutron-induced fission yields, implantation foils were located at different positions in the ion guide. The gamma spectra from these foils and the fission targets are compared to the results from the GEANT4 simulation.

In order to allow fission yield measurements in the low yield regions, towards the tails and in the symmetric part of the mass distribution, the stopping and extraction efficiency of the ion guide has to be significantly improved. This objective can be achieved by increasing the size while introducing electric field guidance using a combination of static electrodes and an RF-carpet. To this end, the GEANT4 model is used to optimise the design of such an ion guide.
\end{abstract}

\section{Introduction}

In order to determine independent fission yields for neutron-induced fission, a proton-neutron converter [1] and an ion guide [2] in which fission fragments are produced and collected has been developed at the IGISOL facility [3] at the University of Jyväskylä, Finland. A measurement campaign of neutron-induced fission yields was conducted in 2016. In this experiment, a $\beta-\gamma$ spectroscopy station was used to identify the fission products (FPs). Details and results from that experiment can be found in the paper by Mattera et al. [4].

The above-mentioned measurement demonstrated a need to increase the extracted yield of FPs. This can be achieved either by improving the proton-neutron conversion ratio of the pn-converter or the collection effiency of the ion guide. In this article we focus on the benchmark of a simulation model for the ion guide which has been developed with the aim of improving the collection efficiency.

Some simulations $[5,6]$ on FPs in the ion guide have already been reported. A GEANT4 model, identical to the setup used in the measurement (Figure 1), has been developed [6]. In the experiment for neutron-induced fission, spectroscopy measurements of implantation foils that were located in the ion guide were conducted after the proton beam was turned off, but the data has not been analyzed until now. On the one hand, FPs stopped in those implantation foils would emit $\gamma$ rays during the measurement peri-

\footnotetext{
*e-mail: zhihao.gao@physics.uu.se

**e-mail: andreas.solders@physics.uu.se
}

ods whose counts represent the yields of FPs. On the other hand, the expected count rates of $\gamma$ rays from implanted FPs in the foils could be obtained from the GEANT4 simulation and compared with the experiment. By comparing the calculated number of $\gamma$ rays with the counts of $\gamma$ rays extracted from the spectra, the simulation could be benchmarked. Based on the benchmark, the GEANT4 model could be corrected and used to optimise a new design of the ion guide.

\section{Spectroscopy measurement}

To know the production of FPs in the ion guide, the implantation foils and targets (see Figure 1) were removed and measured with a HPGe detector in a low-background area after the beam was turned off.

\subsection{Setup}

Figure 1 shows the cross sectional schematics of the ion guide which was used in the experiment in 2016. A neutron field is produced by a proton beam at an energy of $29.6 \mathrm{MeV}$ impinging on a pn-converter target (Be). Fissions are induced by the neutron flux within the uranium targets (red) which are attached to aluminum supports (black). Some of the FPs escape the targets and are thermalised and stopped by the helium gas (purple), or in the aluminum backings (black). Some FPs could pass through the helium gas and are stopped in the titanium foil (blue) or the walls of the ion guide. 


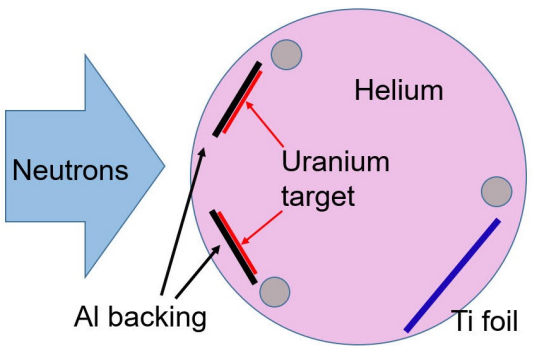

Figure 1. Cross sectional schematics of the ion guide used in the experiment in 2016. Grey circles: rods between the two end caps.

\subsection{Measurements}

When the proton beam was turned off, the foils inside the ion guide were removed and transported to the lowbackground station. The foils were placed close to the HPGe detector at a distance of $16 \mathrm{~cm}$ and measured separately. The foils and the HPGe detector were surrounded by lead. The order, length of measurement time and dead time for these foils are listed in Table 1.

Table 1. Schedule of measurements. Top and bottom refer to upper and lower position in Figure 1.

\begin{tabular}{lll}
\hline Measurement & Duration & Dead time \\
\hline Beam on & $2.5 \mathrm{~d}$ & $*$ \\
\hline Cooling down & $5.22 \mathrm{~d}$ & $*$ \\
\hline Uranium at bottom & $9.8 \mathrm{~h}$ & $6.95 \%$ \\
\hline Uranium at top & $8.9 \mathrm{~h}$ & $6.48 \%$ \\
\hline Cooling down & $2.14 \mathrm{~d}$ & $*$ \\
\hline Ti foil & $24 \mathrm{~h}$ & $0.22 \%$ \\
\hline Al backings & $24 \mathrm{~h}$ & $0.31 \%$ \\
\hline Al backing at top & $24 \mathrm{~h}$ & $0.18 \%$ \\
\hline Al backing at bottom & $32 \mathrm{~h}$ & $0.19 \%$ \\
\hline Ti foil & $24 \mathrm{~h}$ & $0.18 \%$ \\
\hline Ti foil & $16 \mathrm{~h}$ & $0.17 \%$ \\
\hline
\end{tabular}

From the analysis of the spectroscopy data, $37 \gamma$ transitions were found from which 11 mass chains could be derived. Figure 2 shows an example for mass number $\mathrm{A}=95$. This mass chain could be confirmed because three $\gamma$ transitions are found in the spectra.

Energy and efficiency calibration of the detector was done with a ${ }^{133} \mathrm{Ba}$ source and a source mixed of ${ }^{241} \mathrm{Am}$, ${ }^{137} \mathrm{Cs}$ and ${ }^{60} \mathrm{Co}$. Taking the efficiency into account, the actual counts of $\gamma$ rays $\mathrm{N}_{e}$ are extracted from the spectra. $\mathrm{N}_{e}$ will be compared with the results from the simulation.

\section{Simulation}

The simulation model that combines MCNPX [7], GEF [8] and GEANT4 [9] has been extensively described elsewhere [6]. MCNPX is used to simulate the neutron flux that is produced from the pn-converter [1]. GEF estimates

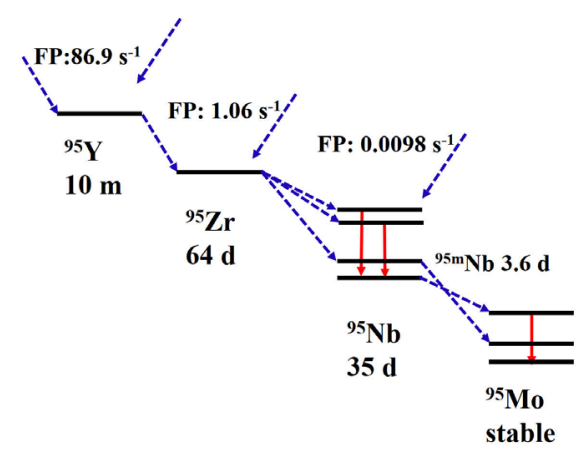

Figure 2. Decay chain with mass number $A=95$. This mass chain is confirmed because $3 \gamma$ transitions, shown in red, were detected.

the yields of the fission fragments that are generated in the uranium targets. The main simulation code based on GEANT4 is responsible for the transport of FPs in the ion guide.

In the simulation, the fission position is sampled uniformly in the uranium targets and the neutron energy is sampled uniformly from $0 \mathrm{MeV}$ to $30 \mathrm{MeV}$. Mass number (A) and charge number ( $\mathrm{Z}$ ) of the FPs are extracted from fission yield distributions obtained from GEF for each integer neutron energy while the the kinetic energy is sampled from the energy distribution of the corresponding isobar. In this manner, a single (not two) fission fragment is generated per fission event. Finally, to obtain absolute numbers, the results are weighted with the fission rate.

\subsection{Analysis}

In one run of the GEANT4 simulation, $10^{8}$ events (post neutron fission products) are generated. The weight comes from the fission rate which depends on the cross section and the neutron flux energy distribution at the given position. The sum of weights for all FPs in the simulation corresponds to the expected total number of fission products (double fission rate) during one second when the proton beam is on with a current of $10 \mu \mathrm{A}$.

The weight for one event (FP) is calculated as Weight $=2 \times$ FissionRate $/ 10^{8}$. The factor 2 comes from the fact that only one FP is generated per fission in the simulation. The fission rate is calculated by $\mathrm{FR}=$ $I_{p} \times \sigma_{f}\left(E_{n}\right) \times N_{U} \times \phi\left(E_{n}, \bar{r}\right) \times 30$, where $I_{p}$ represents the beam current of $10 \mu \mathrm{A} . \sigma_{f}\left(E_{n}\right)$ is the microscopical cross section for fission (taken from the ENDF/B-VII.1 evalution [10]). $N_{U}$ is the number of uranium atoms of the targets and $\phi\left(E_{n}, \bar{r}\right)$ is the neutron flux obtained from MCNPX. The factor of 30 is the number of bins of neutron flux from MCNP. The sum of weights for one isotope corresponds to the yield per second of that FP.

\subsection{Calculation}

Through the weighting process, the FY of each nuclide from the simulation is calculated. FPs were stopped in different positions: the uranium targets, the aluminum back- 
ings and the titanium foil. Summing up the yields of FPs stopped in the respective foil, we could derive how many FPs were implanted in that particular foil in one second. Based on the production rate of FPs implanted in each foil, the build-up and decay of every FP could be calculated. Three time periods have to be mentioned, beam on, cooling down and spectroscopy measurements.

In the calculation, one minute is chosen as the step length. For every decay chain, any production of nuclides with half-lifes shorter than one minute is added to that of the daughter nucleus. If the half-life of the nucleus is longer than one minute, it could be built up in the calculation. In each minute when the beam is on, the number of a particular FP $\left(\mathrm{N}_{F P}\right)$ is increased by the sum of $F Y \times 60$ and the number of the decayed precursors $\lambda_{\text {pre }} \times 60 \times N_{F P_{p r e}}$. Meanwhile, $\mathrm{N}_{F P}$ is decreased by decay $\lambda \times 60 \times N_{F P}$, where $\lambda$ is the decay constant of the FP. So the number of a FP $\left(\mathrm{N}_{F P}^{m}\right)$ during beam on is calculated by:

$N_{F P}^{m}=F Y \times 60-\lambda \times 60 \times N_{F P}^{m-1}+N_{F P}^{m-1}+\lambda_{p r e} \times 60 \times N_{F P_{p r e}}^{m-1}$

and when the beam was turned off,

$$
N_{F P}^{m}=-\lambda \times 60 \times N_{F P}^{m-1}+N_{F P}^{m-1}+\lambda_{\text {pre }} \times 60 \times N_{F P_{p r e}}^{m-1}
$$

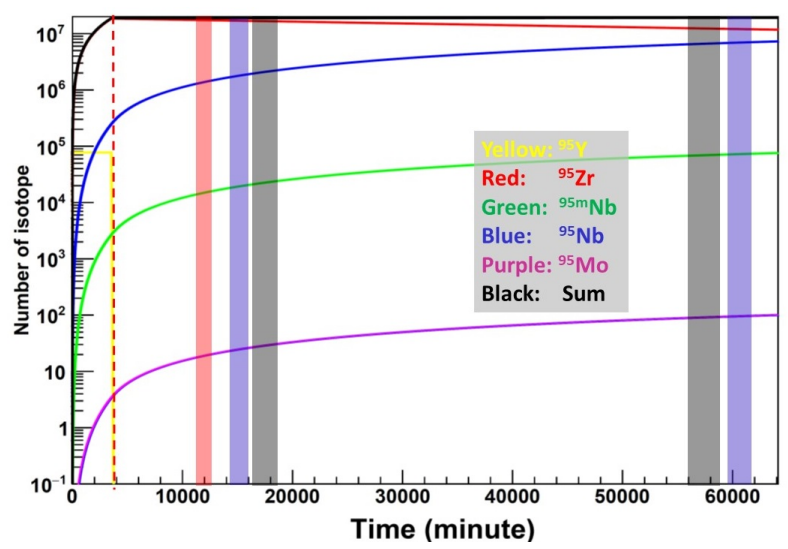

Figure 3. Evolution of the build-up and decay of the $A=95$ chain. To the left of the red dashed line the beam was on. Red region: Measurement of uranium targets. Blue regions: Measurements of titanium foil. Black regions: Measurements of aluminum backings.

Figure 3 shows the build-up and decay process of the decay chain with mass number 95 . In the picture, colored regions correspond to spectroscopy measurements of the respective foils. During the measurement time, the number of the decayed FPs per minute could be calculated by $\lambda \times 60 \times N_{F P}$ and are added up to $\mathrm{N}_{\text {decay }}$. The expected number of each $\gamma$ ray $\left(\mathbf{N}_{s}\right)$ is calculated by multiplying $\mathbf{N}_{\text {decay }}$ with the tabulated branching ratio [11]. This could then be directly compared with the results of the experiment $\mathrm{N}_{e}$.

\section{Comparisons and Discussions}

The numbers of $\gamma$ rays $\left(N_{e}\right)$ are extracted from the $\gamma$ spectroscopy data and the expected numbers of $\gamma$ rays $\left(N_{s}\right)$ based on the GEANT4 simulation are obtained by the calculation above. A ratio $R=N_{s} / N_{e}$ is defined to build a relationship between the simulation and the 2016 experiment. $\mathrm{R}$ should equal one if the simulation is identical to the experiment.

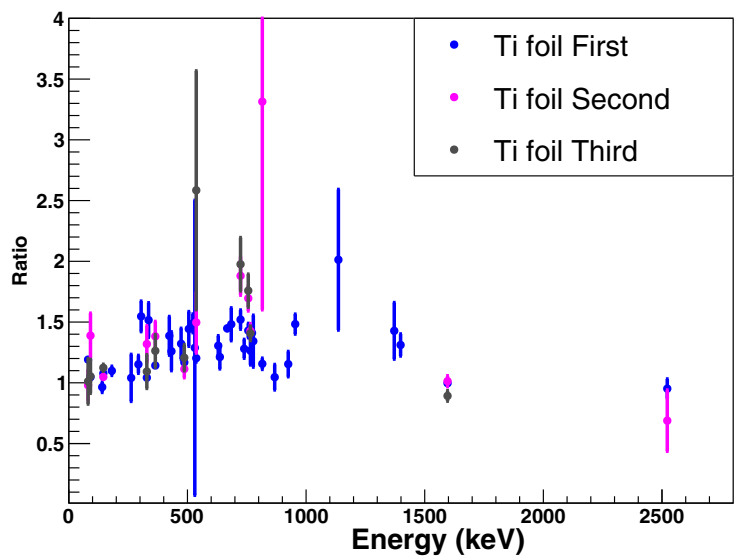

Figure 4. Ratios from three measurements of the titanium foil at different times show an agreement.

Figure 4 shows the ratios from measurements of the titanium foil that were conducted at three different times. The data from the three $\gamma$-spectroscopy measurements show a consistent behaviour. This suggests that the energy calibration did not change and no other problem occured during the measurement.

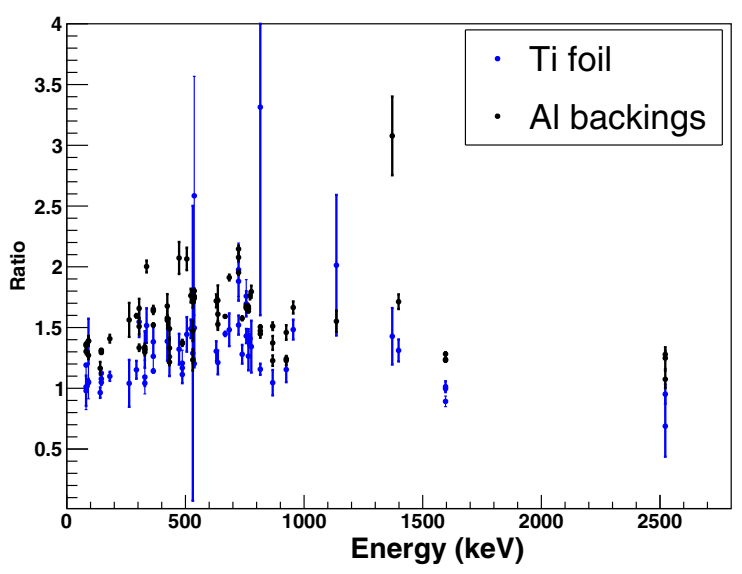

Figure 5. Ratios from the titanium foil and aluminum backings. Data points come from all the measurements of titanium and aluminum foils.

As shown in Figure 1 there is a helium gas between the uranium targets and the titanium foil, while nothing hinders FPs from the uranium targets to reach the aluminum backings. The difference between the ratios from the two foils could represent the stopping power of the helium gas. In Figure 5, ratios from the aluminum backings are slightly higher than that from the titanium foil. It means that more FPs pass through the helium gas and reach the titanium 
foil in the experiment compared to the simulation. This indicates that the stopping power of the helium gas in the experiment is lower than that in the simulation.

Another fact in Figure 5 is that most ratios are larger than one. However, all ratios should be one if the simulation is identical to the experiment. This deviation will be discussed by coupling Figure 5 and 7.

To see the stopping power of the helium gas more directly, a Ti/Al ratio is calculated by dividing the number of $\gamma$ rays from the first measurement of titanium foil with that from the first measurement of aluminum backings. This ratio is related to the stopping efficiency of the helium gas. Actually, the measurements of the two foils were conducted at different times. In the simulation, the number of $\gamma$ rays is calculated in the corresponding period for two foils. The deviation resulting from the difference in measurement time is shown in Figure 6. The corrected Ti/Al ratio is calculated by $\mathrm{N}_{s}$ from the titanium foil over $\mathrm{N}_{s}$ from the aluminum backings which is derived in the first measurement of the titanium foil. We could not correct the $\mathrm{N}_{s}$ from the aluminum backings obtained from the experiment because we do not know the real independent yields of FPs in the experiment.

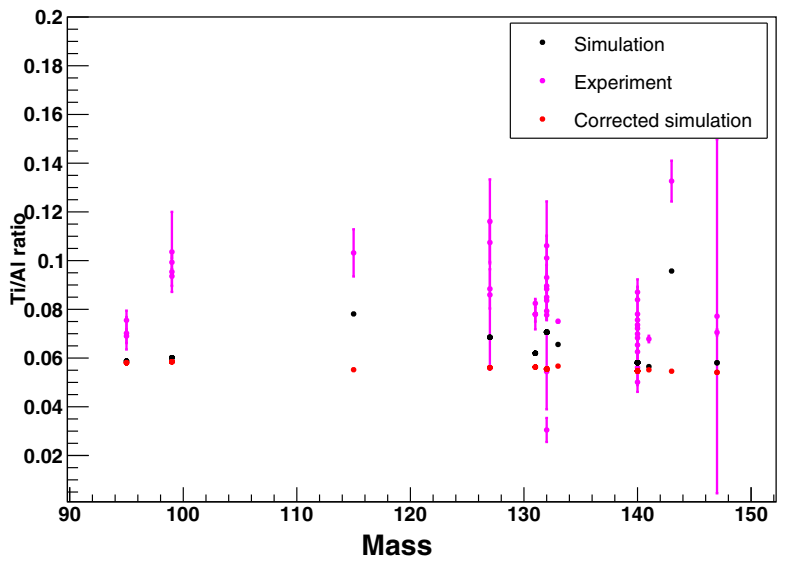

Figure 6. Purple Ti/Al ratios: number of $\gamma$ rays from the first measurement of titanium foil over that from the first measurement of aluminum backings. Black Ti/Al ratios: calculated Ti/Al ratios from the simulation. Red Ti/Al ratios: corrected Ti/Al ratios during the measurement period of titanium foil in the simulation.

Comparing the results from the simulation and the experiment, Figure 6 indicates that the Ti/Al ratios from the experiment are slightly higher than that from the simulation. This agrees with the conclusion above, that the real stopping efficiency of the helium gas is slightly lower that what is used in the simulation.

The reason for the lower stopping efficiency of the measurement compared to the simulation could be due to the uncertainty of the helium gas pressure inside the ion guide. This pressure is not measured inside the guide but is estimated from the flow and pressure of the gas in the feed line. In other words, the simulation seems to give consistent results.

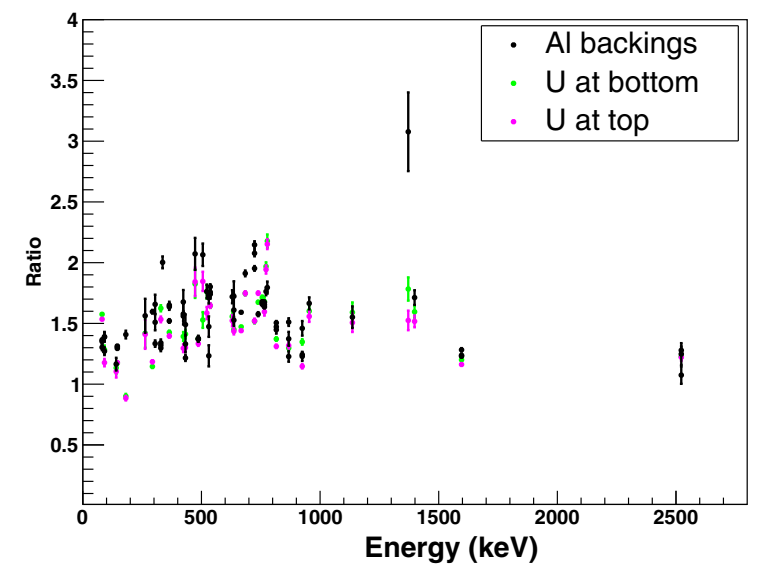

Figure 7. Ratios from the uranium targets and aluminum backings.

Two reasons may lead to the fact that most ratios are higher than one. One is that more FPs escape from the uranium targets in the simulation than in the experiment. Another is that more fissions are generated inside the uranium targets in the simulation than in the experiment. To explore these possibilities, the ratios from the aluminum backings are compared to those from the uranium targets. As Figure 7 shows, the data from the uranium targets also are higher than one and agree rather well with that from the aluminum backings. This indicates that the transport of FPs from the uranium targets to the aluminum backings in the simulation is correct. Hence, the reason for the deviation from one in Figure 5 and 7 is that more fissions are generated in the simulation than in the experiment. This point to problems with the MCNPX simulation of the neutron production.

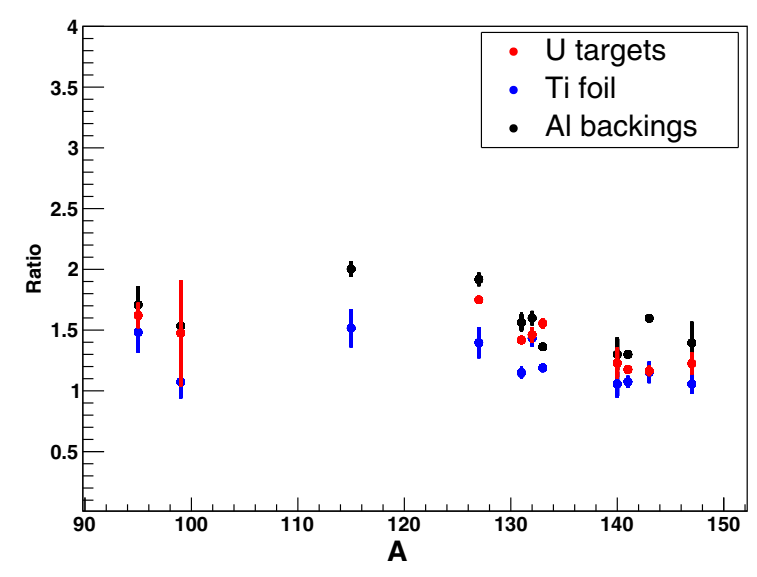

Figure 8. Merged ratios by weighted uncertainties from the three foils versus mass number.

In order to look for a possible mass dependency of the stopping power of the helium gas, the ratios of different $\gamma$ transitions from the same isobar are merged by weighting with the uncertainties. The result is shown in Figure 8 
where no clear mass dependency within the experimental uncertainties can be observed.

If the simulation is identical to the experiment, the ratios from the uranium targets and aluminum backings should be equal to one. The overall deviation between simulation and experiment was determined by calculating the weighted average ratio for all masses. For the uranium targets, aluminum backings and the titanium foil, the weighted average values are $1.37 \pm 0.18,1.39 \pm 0.26$ and $1.16 \pm 0.15$ respectively. The values for the uranium targets and the aluminum backings are almost same as they are positioned close to each othe. Their average value is $1.38 \pm 0.14$, indicating that the fission rate in the simulation is overestimated about $38 \%$.

The difference between the aluminum backings $(1.39 \pm 0.26)$ and the titanium foil $(1.16 \pm 0.15)$ is about $15 \%$. A probable explanation for this discrepancy could be that the helium gas pressure assumed in the simulation is higher than the actual pressure in the ion guide. However, in order for the simulation to reproduce the result of the measurement to equal the value (1.38) the pressure has to be reduced from the nominal 400 mbar to 220 mbar. Such a decreas seems to be unreasonably large, hence, other reasons for the discrepancy should be investigated. For example, the uncertainties from the positions of the uranium targets and titanium foil that determines the neutron flux, fluctuation of the current of proton beam and other systemic uncertainties.

To investgate reasons for the deviation of the fission rate, the neutron flux used in the simulation should be benchmarked. For this reason three foils for neutron activation that are mounted between the pn-converter and the ion guide were measured with the HPGe detector and analysis in ongoing. Also the titanium foil located in the ion guide was activated by $(\mathrm{n}, \mathrm{p})$ reactions, and $\gamma$ transitions from several scandium isotopes were identified. This information, together with data from dedicated neutron activation foils, will be used to estimate the neutron flux to compare with the MCNPX simulations.

\section{Conclusions and outlook}

The GEANT4 model has been benchmarked by comparing the results from the simulation with the experimen- tal data. The comparisons indicate that the fission rate in the simulations is overestimated with approximately $38 \%$. Further analysis of neutron activation foils is ongoing in order to verify the fission rate. Furthermore the simulations suggest that the helium gas pressure in the ion guide is substantially lower than the nominal $400 \mathrm{mbar}$, as more fission products than expected reach the foil far away from the fission target.

Overall, the ion transport in the GEANT4 model is verified within uncertainties. Mass dependency of the stopping efficiency of the helium gas is not observed. Once fully benchmarked the simulation model will be used to optimize the design of a larger, more efficient ion guide using electric field guidance.

\section{References}

[1] A. Mattera et al., European Physics Journal A 53, 173 (2017).

[2] D. Gorelov, H. Penttilä, A. Al-Adili et al., Nucl. Instrum. Methods Phys. Res. B 376, 46 (2016).

[3] I. D. Moore et al., Nucl. Instrum. Methods Phys. Res. B 317, 208 (2013).

[4] A. Mattera, et al., European Physics Journal A 54, 33 (2018).

[5] A. Al-Adili et al., European Physics Journal A 51, 59 (2015).

[6] K. Jansson, et al., European Physics Journal A 53, 243 (2017).

[7] G.W. McKinney, et al., MCNPX 2.5.0 - New Features Demonstrated, in Proceedings of the MC2005 Conference, Chattanooga, Tennessee, April 17-21, 2005 (American Nuclear Society, 2005) LA-UR-04-8695.

[8] K.-H. Schmidt, et al., Nucl. Data Sheets 131, 107 (2016).

[9] S. Agostinelli et al., Nucl. Instrum. Methods A 506, 250 (2003).

[10] M.B. Chadwick et al., Nucl. Data Sheet 112, 2887 (2011)

[11] https://www.nndc.bnl.gov/nudat2/ 